IMTC 2005 - Instrumentation and Measurement

Technology Conference

Ottawa, canada, May 17-19, 2005

\title{
Introduction of a 2 transducer ultrasonic mass flow meter
}

\author{
J. van Deventer \\ Department of Computer Science and Electrical Engineering, \\ Luleå University of Technology, \\ 97187 Luleå, Sweden \\ Phone: +46 (70) 679 1336, Fax: +46 (920) 4920 82, E-mail: deventer@1tu.se.
}

\begin{abstract}
This article presents a new ultrasonic mass flow meter that has only two transducers. The mass flow is inferred from volumetric flow and density. Each of the transducers is an ultrasonic densitometer based on a design by Lynnworth. The flow is estimated by the transit time difference of the sound pulse between the up and downstream acoustic propagation. Beyond presenting the meter, this article does looks at its signal at zero flow to address the issue of reciprocity in transit time flow meters which is in contradiction with the theory of transit time flow meters.
\end{abstract}

Keywords - Ultrasonic, mass flow meter, densitometer, reciprocity, zero flow

\section{INTRODUCTION}

Measuring mass flow becomes important when the density of a liquid varies such that volumetric flow information is not sufficient anymore. As an example, consider a process industry where mouthwash is the product; water, glycerin and alcohol are mixed together with the proper proportions. If the glycerin or alcohol have absorbed water, volumetric flow meters would not track that while mass flow meters would.

Commercially available mass flow meters are based on different concepts. One can divide them into two groups: direct and indirect measurement of mass flow. A Coriolis mass flow meter measures directly the mass flow from the deformation of an oscillating pipe. On the other hand, an ultrasonic flow meter belongs to the second category where the mass flow is inferred from the product of a measurement of the volumetric flow and a measurement of the liquid density. These two measurements were made from separate units resulting in a 3 ultrasonic transducer system [1] or in a 4 ultrasonic transducer system [2]. The current mass flow meter is the first to use only 2 ultrasonic transducers. Each of them is able to measure the acoustic impedance of the liquid while together they are able to measure the flow velocity and the speed of sound in the liquid to determine the density of the liquid.

The concept of measuring liquid mass flow using ultrasound is not new and patents towards that goal have been awarded since the 1950 's. However, there are several issues that complicate the accurate measurement of the liquid's density. The method used with the current transducer is based on the reflection of sound as the acoustic pulse enters the liquid from the probe. The amount of sound reflected is an indication of the liquid's acoustic impedance. The characteristic acoustic impedance $z$ of a medium reduces mathematically to

$$
z=\rho c
$$

where $\rho$ is the density of the medium and $c$ is the speed of sound through that medium. One problem with this method is that if the incident pulse's amplitude changes, then so does that of the echo. It becomes therefore essential to monitor the amplitude of the emitted pulse. Various solutions have been proposed to address this which include a thin film receiver across the probe [3], a reflective backing [4], or a reference interface through the probe [5]. The solution chosen here is the one proposed by Lynnworth [1] as it is stable with respect to changes in temperature since it does not have glued interfaces. Another problem occurs with changes in temperature, which results in changes of the characteristics of the probe material as density, speed of sound and mostly acoustic absorption. This has been addressed by recognizing that the temperature influences the time of flight throughout the probe resulting from changes in speed of sound through the probe[5]. Therefore the temperature can be estimated and the acoustic attenuation deduced and the density corrected. A third issue is the contamination deposit layer that can alters the reflection and transmission of sound at the probe-liquid interface just as does lens coating for light in a camera. The fact that this coating facilitates the transmission of certain frequencies and not others provides a solution. With the help of simulations, it has been shown that the presence and thickness of the contamination layer can be determined by comparing the coefficient of reflection at different frequencies [7]. The method requires very large bandwidth or the presence of higher harmonics.

The flow meter part relies on the fact that sound needs a medium to travel through and if that medium is moving with respect to a fixed reference point, it will influence the perceived speed of sound. A transit time ultrasonic flow meter estimates the fluid flow velocity from the different times of flight of up and down stream acoustic pulses over the distance parallel to the flow direction. Variations in fluid dynamics between lam- 


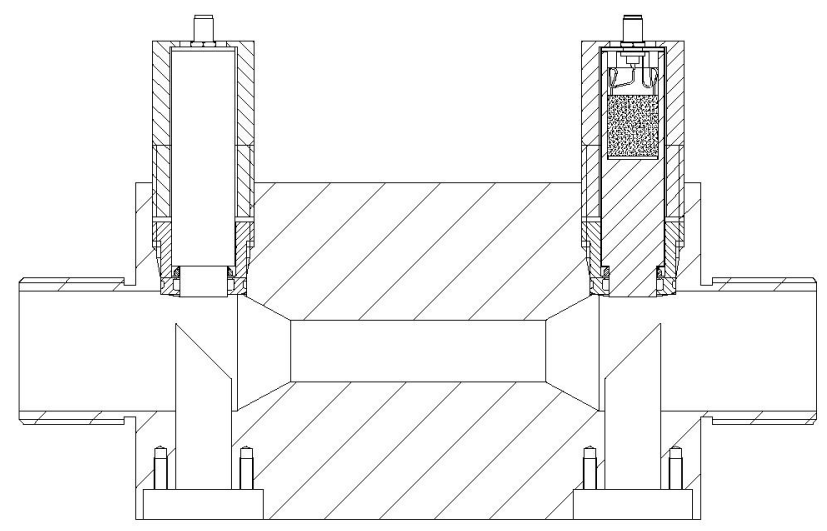

Fig. 1. Cross section of the mass flow meter. The left transducer is complete while the right one is a cross section.

inar and turbulent flows require a correction of the estimated flow. This correction or compensation is obtained from a calibration of the meter. Naturally, viscosity of the fluid is an issue but it could be measured from the frequency dependent attenuation of the acoustic pulses.

One issue of special interest here is the performance a transit time flow meter at zero flow. Since the fluid is not moving the up and downstream times of flight should be identical. Hemp shows analytically that such should be the case [8]. However, experimentally there is a need to correct for that. Simulations of a transit time flow meter showed that this is due to the fact that the transducers are not totally identical [9]. The new mass flow meter offers a new chance to study this since each densitometer receives and echo when the respective acoustic pulses leave the probes for the liquid.

\section{THE MASS FLOW METER}

Figure 1 shows the cross section of the mass flow meter. It is made of an aluminum block. Its inlet and outlet have a diameter of $39 \mathrm{~mm}$ and the reduced part has a diameter of $20 \mathrm{~mm}$. Two brass reflectors redirect the sound beams to the other ultrasonic transducer. The reflectors are inserted at the bottom of figure 1 and their center is separated by $140 \mathrm{~mm}$. The two ultrasonic sonic transducers located at the top are identical and both capable of estimating the acoustic impedance of the liquid.

The densitometer probes are made of polyetheretherketone (PEEK) polymer rods with a diameter of $20.8 \mathrm{~mm}$. The ultrasonic sensing elements are $16 \mathrm{~mm}$ in diameter $\mathrm{Pz}-27$ piezoceramic disks with a center frequency of $1 \mathrm{MHz}$ and are from Ferroperm, Denmark. An absorbing tungsten powder filled Araldite epoxy backs the disks. The epoxy consists of $20 \%$ by weight of the tungsten. The probe-liquid interface is located $45 \mathrm{~mm}$ in front of the piezoceramic disks. $10 \mathrm{~mm}$ prior to this interface with the liquid, the probe's diameter is reduced to

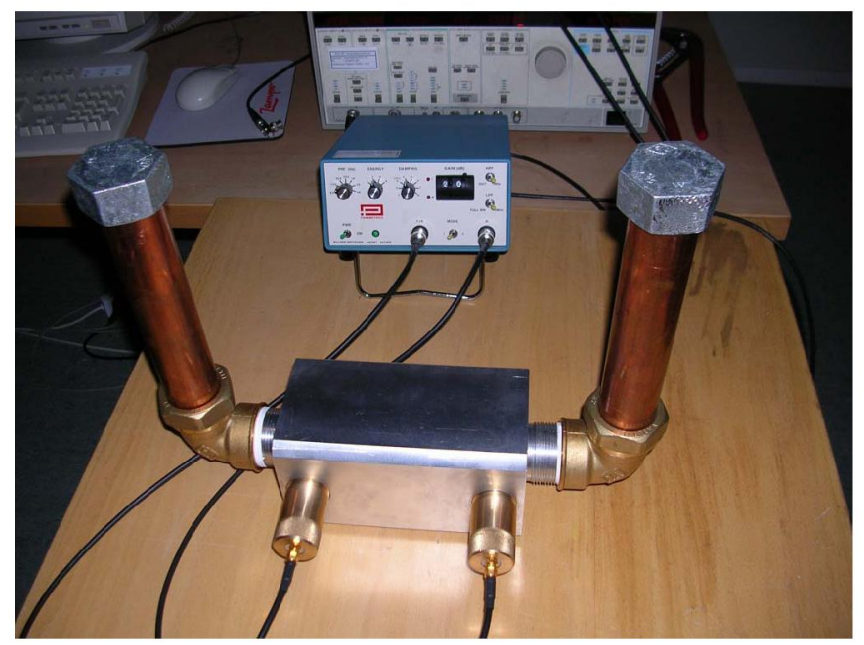

Fig. 2. The mass flow meter with the two transducers at the bottom of the picture.

$16.5 \mathrm{~mm}$. From this step, which creates an interface with air, is reflected the outer ring of the acoustic beam. The echo is the reference of the emitted pulse. The probe is based on a design by Lynnworth used in a three ultrasonic sensors mass flow meter [1]. Each densitometer receives two echoes (reflected pulses) to calculate the acoustic impedance of the liquid and a pulse from the other probe to calculate the speed of sound in the liquid and the flow velocity of the liquid.

The two echoes $A_{1}$ and $A_{2}$ are measured and related to the emitted impulse $A_{0}$ as

$$
A_{1}=K \frac{z_{a}-z_{p}}{z_{a}+z_{p}} e^{-2 \alpha_{p} d_{1}} A_{0},
$$

and

$$
A_{2}=(1-K) \frac{z_{l}-z_{p}}{z_{l}+z_{p}} e^{-2 \alpha_{p}\left(d_{1}+d_{2}\right)} A_{0} .
$$

Where $z_{a}$ is the acoustic impedance of air, $z_{p}$ that of the probe and $z_{l}$ that of the liquid which we are tying to measure. $\alpha_{p}$ is the coefficient of acoustic attenuation within the probe. $d_{1}$ is the distance separating the piezoceramic disc and the step while $d_{2}$ is the distance separating the step and the end of the probe that is in contact with the liquid. $K$ is a correction factor as not the whole beam's cross section is reflected but only its outer ring in the case of $A_{1}$ and its center in the case of $A_{2}$. Since $A_{0}$ can vary due to changes in temperature, aging or excitation from the electronics, it can be eliminated by combining the two equations. The only unknown then is $z_{l}$ for which we can solve for. As stated in the introduction, the constants $z_{p}, \alpha_{p}$, $d_{1}$ and $d_{2}$ are temperature dependent and can be estimated via the acoustic times of flight.

To obtain the velocity of sound through the liquid and the flow velocity, information from both transducers have to be combined. To refer to the pulses correctly we use the following notation for the arrival times: $t_{x y}$ is the time of the $x^{\text {th }}$ pulse 

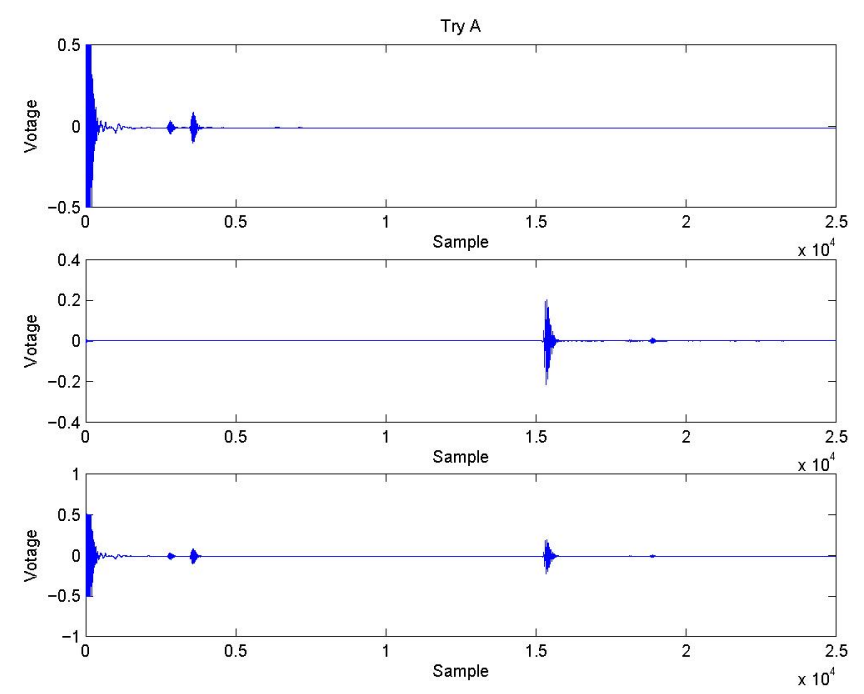

Fig. 3. Signal received by the emitting transducer (top), signal received by the receiving transducer (middle) and their combination (bottom),

is received by the $y^{\text {th }}$ transducer. Such nothation can also be used for the echoes in equations (2) and (3). $d_{l}$ is the length of the acoustic path in the liquid. The average of the transit times between the second and third echoes is required to obtain the velocity of sound through the liquid.

$$
c_{l}=\frac{2 d_{l}}{\left(t_{3 b}-t_{2 a}\right)+\left(t_{3 a}-t_{2 b}\right)} .
$$

The flow velocity $v$ is obtained from the difference between those times

$$
v=\frac{d_{m}}{\left(t_{3 b}-t_{2 a}\right)-\left(t_{3 a}-t_{2 b}\right)},
$$

where $d_{m}$ is the distance separating the reflector which in this case is $140 \mathrm{~mm}$. It is the distance over which the flow influences the times of flight.

\section{RESULTS}

Figure 2 shows the assembled mass flow meter with extension tubes to fill the whole system with water. The transducers and reflectors are kept horizontal to minimize the influence of deposits or air bubbles on the measurements. The mass flow meter is connected to a Panametrics 5073 Pulser-Receiver whose output is sampled by a Tektronix RTD710 at a frequency of $100 \mathrm{MHz}$. This setup allows only one of the transducer to be fired and the received signals have to be done two times. Once to record the echoes or reflected pulses by the emitting probe while the second time to record the transmitted pulse.

The top plot of Figure 3 shows the received echo from the emitting probe or liquid acoustic impedance meter. The middle plot shows the received signal by the receiving transducer.

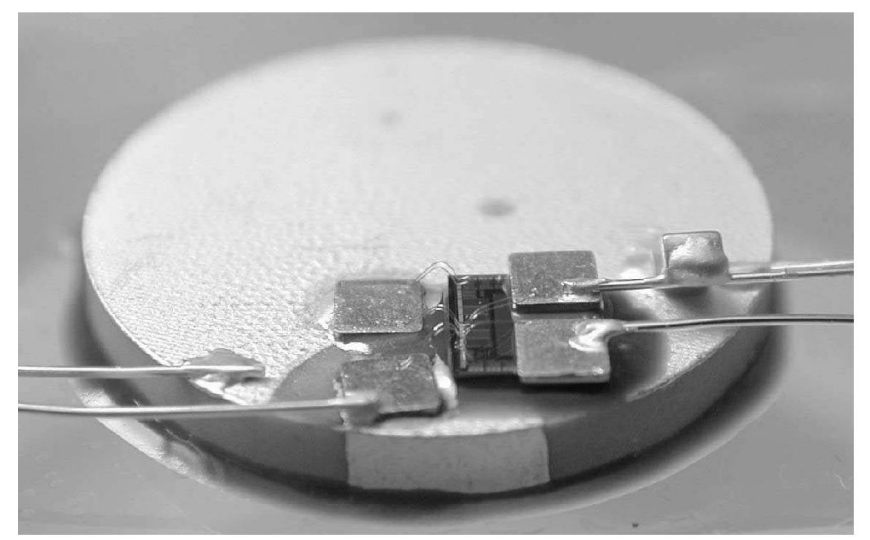

Fig. 4. Electronics for the future densitometers to minimize transit time uncertainty at zero flow. The application specific integrated circuits (ASIC) is glued onto the piezoceramic disk.

Their combination is shown in lower plot of the figure. If there is no liquid flow, the information is sufficient to estimate the density of the liquid between the reflector where the flow carries or retards the acoustic pulses. Oscillating flow with the current set up is possible by comparing the time of flights of the third echo with a reference signal recorded in a static condition.

A setup to provide transit time flow meter was also evaluated. Two Panametrics pulser-receivers where used in trans$\mathrm{mit} /$ receive mode. A common signal generator triggered them both externally and their output recorded on each of the oscilloscope's channels. This enabled the reception of the two echoes and the transmitted pulse by both transducers. However, since the two pulser-receivers were of different models, the performance resulted in too much difference in the excitation pulses. The problem to be addressed here is the error at zero flow, i.e. when there is no flow. The time of arrival of the echoes and pulses are not the same for the received signals from the two probes. One can quickly justify the difference in time of arrival of the reflected echoes since they are from two different probes. The pulses are received from the opposite transducers should arrive at the same time because the pulses have traveled through the exactly the same media, just in opposite directions. This is in contradiction to the theory of transit time flow meters [8].

\section{CONLUSION AND FUTURE WORK}

We introduced a new ultrasonic mass flow meter and showed the signal received with a standard pulser-receiver. Current efforts include the incorporation of electronics into the probes. Building all the associated electronics into application specific integrated circuits (ASIC) minimizes variations as they are from silicon disk. Figure 4 shows such an ASIC glued onto a piezoceramic disk built at Luleå University of Technology. 


\section{REFENCES}

[1] L. C. Lynnworth, N. E. Pedersen, E. N. Carnevale, "Ultrasonic Mass Flowmeter for Army Aircraft Engine Diagnostics", National Technical Information Service, Springfield, VA, 1973.

[2] A. R. Guilbert and M. L. Sanderson, "A high accuracy ultrasonic mass flowmeter for liquids", FLOMEKO 96 proceedings, pp. 244-249.

[3] J. C. Adamoski, F. Buiochi and R. Sigelmann, "Ultrasonic measurement of density of liquids flowing in tubes", IEEE Trans. Ultrason., Ferroelec., Freq. Contr, vol. 45, no. 1, pp. 48-56, 1998.

[4] A. Püttmer, Ultrasonic Density Sensor for Liquids., Ph.D. Thesis, Shaker Verlag, Aachen, 1998, ISBN 3-8265-4614-8.

[5] J. van Deventer, J. Delsing, "Thermostatic and dynamic performance of an ultrasonic density probe", IEEE Trans. Ultrason., Ferroelect., Freq. Contr., 3 (48), pp. 675-682 2001.

[6] D. W. Spizter, editor, "Flow Measurement", Instrument Society of America, Research Triangle Park, NC, 1991.

[7] J. van Deventer, "Detection of, and compensation for error inducing thin layer deposits on an ultrasonic densitometer for liquids," Instrumentation and Measurement Technology Conference, 2003. IMTC '03. Proceedings of the 20th IEEE, 2003, pp. 648-651.

[8] J. Hemp, Theory of transit time ultrasonic flowmeters, Journal of sound and vibration, 84 (1) (1982) 133-147.

[9] J. van Deventer; J. Delsing, "Apparent transducer non-reciprocity in an ultrasonic flow meter," in Ultrasonics International 2001, 2002, pp. 403405 . 